\title{
PT1 Stage Finding
}

National Cancer Institute

\section{Source}

National Cancer Institute. pT1 Stage Finding. NCI Thesaurus. Code C48759.

A pathologic primary tumor TNM stage finding. The definition of PT 1 stage finding depends on the particular type of cancer that it refers to; for example, for breast cancer, PT 1 stage finding is defined as follows: cancer with tumor size $2.0 \mathrm{~cm}$ or less in greatest dimension; for colorectal cancer, PT 1 stage finding is defined as follows: cancer with invasion into the submucosa. (from AJCC 6th and 7th Eds.) 